\title{
GBPs coordinate vesicular trafficking for host defence
}

Interferon- $\gamma(\operatorname{IFN} \gamma)$ has a crucial role in immunity against intracellular pathogens. Approximately 2,000 host genes are induced by this cytokine but the functions of many of these genes remain uncharacterized. A study published in Science examined the function of a family of IFN $\gamma$ inducible $65 \mathrm{kDa}$ guanylate-binding proteins (GBPs) and found that these GTPases promote IFN $\gamma$-mediated host defence against intracellular bacterial infections by regulating oxidative responses and bacteriolytic peptide generation.

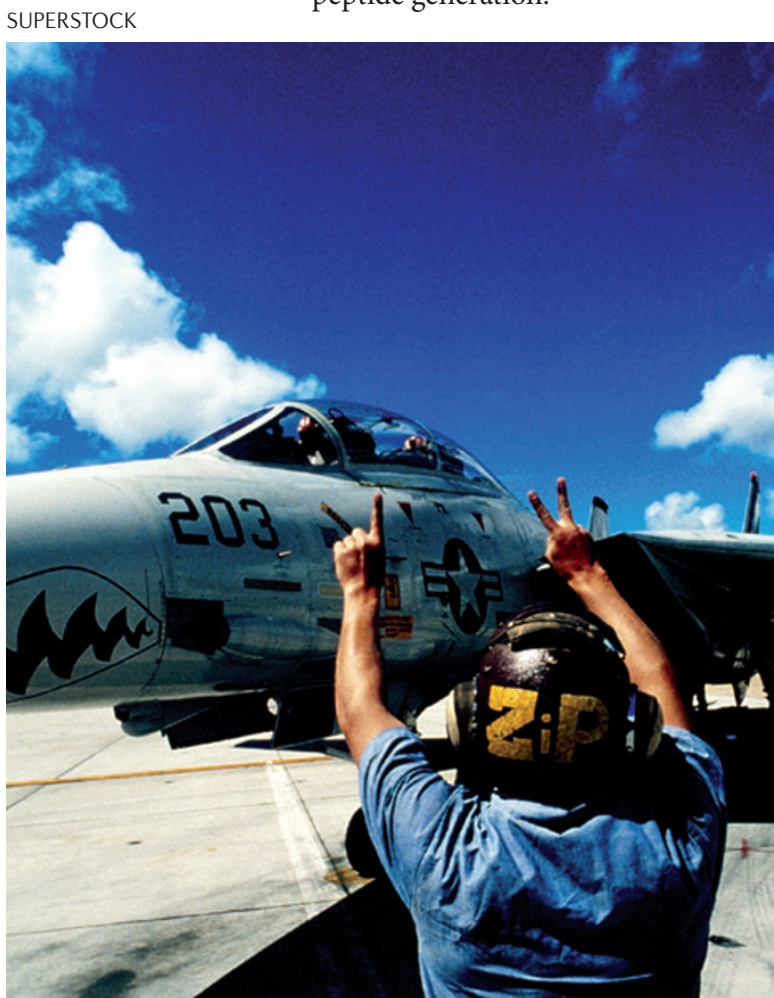

The authors used three different approaches (namely, small interfering RNA (siRNA)-mediated gene silencing, expression of mutant GBPs and gene deletion) to inhibit various GBP family members in macrophages infected with Listeria monocytogenes or Mycobacterium bovis BCG. They found that inhibition of GBP1, GBP6, GBP7 and GBP10 reversed IFN $\gamma$-mediated reduction of bacterial replication, suggesting a role for these GTPases in IFN $\gamma$-mediated killing of intracellular pathogens. Further evidence to support this was provided by the observation that infection of $G b p 1^{-/-}$mice resulted in severe listeriosis or M. bovis BCG-induced morbidity owing to massively increased bacterial replication in the target organs, whereas wild-type mice could control the infection.

But how do these GBPs contribute to IFN $\gamma$-induced bactericidal activities in macrophages? Following infection of macrophages, bacteria are sequestered inside phagosomes, and subsequent lysosomal fusion leads to autophagolysosome formation and bacterial killing. All the protective GBPs were shown to translocate to bacteria-containing phagosomes, suggesting that they may help deliver antimicrobial cargo to the bacteria-containing vacuoles. GBP7 was found to help assemble the NADPH oxidase complex on phagosomal membranes by transporting cytosolic components of the complex (p67phox, p47phox and p40phox) to the membrane for assembly with
NOX2. The NADPH oxidase complex is required for oxidative defence, and cells deficient in functional GBP7 had diminished IFN $\gamma$-induced superoxide production.

Further analysis showed that GBP1 binds to the ubiquitinbinding protein p62 (also known as SQSTM1), which delivers monoubiquitylated proteins to autolysosomes for the generation of bacteriolytic peptides that mediate bacterial killing in autophagolysosomes. Inhibition of GBP1 resulted in defective delivery of p62-bound ubiquitylated proteins to $\mathrm{LC}^{-} \mathrm{B}^{+}$ membranes for engulfment and lysosomal delivery. GBP7 was found to regulate the next step, by bringing ATG4B (autophagy-related gene 4B) to this membrane for membrane elongation and closure. This step is necessary to generate the bacteriolytic peptides for bacterial killing.

So, GBP1 and GBP7 mediate IFN $\gamma$-induced bactericidal activity by promoting oxidative killing and delivering bacteriolytic peptides to autophagolysosomes, thereby contributing to protection against bacterial infection. In addition, GBP6 and GBP10 have a role in IFN $\gamma$-mediated killing of intracellular bacteria, although their mechanisms of action remain to be determined.

Olive Leavy

ORIGINAL RESEARCH PAPER Kim, B.-H. et al. A family of IFN- $\gamma$-inducible $65-\mathrm{kD}$ GTPases protects against bacterial infection. Science 332, 717-721 (2011) 